\title{
Las políticas sociales de lucha contra el sinhogarismo en la Unión Europea y España: alcance, efectividad y principales limitaciones y prioridades
}

\author{
Vicente Marbán Gallego
}

Facultad de Ciencias Económicas, Empresariales y Turismo, Universidad de Alcalá vicente.marban@uah.es

\section{Gregorio Rodríguez Cabrero}

Facultad de Ciencias Económicas, Empresariales y Turismo, Universidad de Alcalá gregorio.rodriguez@uah.es

Europako erakundeek eta Europako zenbait herrialdek eragindako bultzada hazi arren, etxegabetasunaren aurkako borrokak rol marjinal bera mantentzen du gizarte-politiken guztizkoan; behintzat eta batez ere, konparazioan hartuta, etxebizitza, enplegu eta gizarte-zerbitzu gisakoak, etxegabetasun-politikekin integratuago beharko liratekenak. Artikulu honen helburua Europako estrategia nagusien irismena, eraginkortasuna, mugak eta lehentasunak aztertzea da; baita Espainiako egoera ere, Europako Batzorderako azterlan baten emaitzetan oinarritzen dena. Espainian eta Europar Batasunean etxegabetasunaren kartografiarekin eta beste politika batzuekiko koordinazioarekin zerikusia duten gaiei erantzun nahi zaie.

\section{GAKO-HITZAK:}

Etxerik gabeko pertsonak, bizitegi-bazterketa, Housing First, gizarte-politika, Europar Batasuna.
Pese al creciente empuje de las instituciones europeas y de algunos países europeos, la lucha contra el sinhogarismo sigue tenido un papel marginal dentro del conjunto de políticas sociales, sobre todo comparado con aquellas con las que debería estar más integrada, como vivienda, empleo y servicios sociales. El objetivo de este artículo es analizar el alcance, efectividad, limitaciones y prioridades de las principales estrategias europeas, incluido el caso español, según los resultados de un estudio realizado para la Comisión Europea. Pretende responder a cuestiones relacionadas con la cartografía del sinhogarismo en España y en la Unión Europea, la coordinación con otras políticas y entre niveles de la Administración, el seguimiento y evaluación de la efectividad de los servicios para personas sin hogar, la innovación en dichos servicios y si existen limitaciones y retos compartidos por los países de la UE en la lucha contra el sinhogarismo.

\section{Palabras clave:}

Personas sin hogar, exclusión residencial, Housing First, política social, Unión Europea. 


\section{Introducción}

El desarrollo de estrategias integradas contra el sinhogarismo en los estados de la Unión Europea (UE) lleva siendo intensamente demandando por las principales organizaciones no lucrativas y las instituciones europeas durante la última década. Así se plasma, por ejemplo, en el Informe Conjunto sobre Protección Social e Inclusión Social del 2010 (Comisión Europea, 2010), la Conferencia Europea sobre Sinhogarismo celebrada en diciembre de 2010 en Bruselas, las Resoluciones del Parlamento Europeo de septiembre de 2011 y enero de 2014 (Parlamento Europeo, 2011, 2014) o en el Paquete de Inversión Social de la UE de 2013 (Comisión Europea, 2013). Más recientemente, el principio 19 del Pilar Europeo de Derechos Sociales también reconoce el derecho a la vivienda y a la atención de las personas sin hogar y de las personas vulnerables para promover su inclusión social. Sucede, no obstante, que este reconocimiento es inocuo si no se plasma en políticas sociales eficaces que afronten realmente el problema del sinhogarismo.

En este texto se presentan los principales resultados de un estudio realizado para la Comisión Europea en el que han participado los autores y donde analizamos la dimensión del sinhogarismo y la efectividad, limitaciones y prioridades de las estrategias europeas contra el sinhogarismo con especial atención al caso español ${ }^{1}$. Con ello pretendemos no solo difundir los resultados de este estudio basado en el aprendizaje mutuo con otros países de nuestro entorno en materia de sinhogarismo, sino también preguntarnos si tras este aprendizaje siguen existiendo limitaciones y retos compartidos por los países de la UE, incluida España. También queremos responder a otras preguntas de investigación como las siguientes: ¿está adecuadamente cartografiado el sinhogarismo en España y en la UE?; ¿las estrategias contra el sinhogarismo están siendo acompañadas de una coordinación efectiva con las políticas integrales de vivienda, empleo y servicios sociales y entre los distintos niveles de la administración?; ¿están generalizados los procesos sistematizados de seguimiento y evaluación de la efectividad de los servicios para personas sin hogar?; ¿se están produciendo avances en la innovación en estos servicios?; y ¿cuáles son sus limitaciones?

${ }^{1}$ Este trabajo forma parte de un contrato de investigación con la Comisión Europea a través de LISER para el análisis de las políticas sociales en España. También ha sido realizado con el apoyo del proyecto PECOTSAS del Plan Estatal de Investigación Científica y Técnica y de Innovación 2013-2016 del Ministerio de Economía, Industria y Competitividad (ref. CSO2017-85486-P).

\section{Dimensión y estrategias europeas de lucha contra el sinhogarismo}

El primer obstáculo para el diseño de estas estrategias es la ausencia de una "cartografía" del sinhogarismo (Pleace et al., 2018). Actualmente, no hay consenso sobre el método de medición y seguimiento del sinhogarismo en Europa. El punto de partida para acordar una definición es la European Typology on Homelessness and Housing Exclusion (ETHOS) ${ }^{2}$ elaborada por Feantsa y consensuada en la Conferencia Europea sobre sinhogarismo de Bruselas en 2010.

Baptista y Marlier (2019)3 ${ }^{3}$, utilizando la clasificación ETHOS light en 35 países europeos constatan cómo las definiciones y formas de medir el sinhogarismo varían sustancialmente entre los países. Dentro de la definición y medición de las personas sin hogar, estos autores señalan que prácticamente todos los países incluyen la categoría 1 de ETHOS light, la mayoría incluye la categoría 2 y en menor medida la 3 , mientras que según se va avanzado a las categorías 4, 5 y 6, estas tienden a ser cada mas vez minoritarias (menos de la mitad incluyeron la categoría 5 y solo 9 países de los 35 analizados, la categoría 6). Como después comentaremos, España considera dentro de la definición de personas sin hogar solo a las categorías 1 a 4 de ETHOS light. Esta heterogeneidad en la definición de las personas sin hogar se traslada claramente a su cuantificación, con lo que no es posible señalar una cifra total de personas sin hogar en la UE. Incluso para la categoría 1 , que es la que aglutina el mayor consenso, no es posible estimar una cifra total, ya que solo el $60 \%$ de los 35 países analizados por Baptista y Marlier (2019) registran datos a nivel nacional. Otros países recogen cifras de las categorías 1 y 2 de manera conjunta y menos de un tercio recoge información sobre la categoría 4.Estas limitaciones se reproducen, aunque en menor medida, en los países de la UE 15. La Tabla 1 refleja el número de personas sin hogar en la UE 15 según las categorías ETHOS light 1 a 4.

Los datos reflejados en la Tabla 1 deben tomarse con cautela al no ser directamente comparables, ya que la metodología y fechas de referencia son heterogéneas entre los países y no todos los países tienen registros sobre personas sin hogar. No obstante, de manera orientativa, en la UE 15 podrían

${ }^{2}$ La tipología ETHOS puede consultarse en el siguiente enlace: 〈http://bit.ly/2nLhqGf〉. Con el fin de simplificar la comparación demoscópica y estadística entre países, Feantsa ha desarrollado una versión "light" de ETHOS (ETHOS light). En ETHOS light se establecen las siguientes seis categorías: categoría 1 (personas viviendo en la calle); categoría 2 (personas viviendo en alojamientos de emergencia, como albergues nocturnos); categoría 3 (personas viviendo en alojamientos temporales para personas sin hogar); categoría 4 (personas viviendo en instituciones penales o sanitarias), categoría 5 (personas viviendo alojamiento no convencionales debido a la carencia de un hogar) y categoría 6 (personas viviendo temporalmente en hogares convencionales con familia y amistades).

3 En el trabajo de estos autores puede encontrarse un desarrollo más detallado de las estrategias europeas de lucha contra el sinhogarismo en 35 países europeos. Los autores de este artículo han participado en este trabajo analizando el caso español. 
Tabla 1. Personas sin hogar en la UE 15 según las categorías ETHOS light 1-4

\begin{tabular}{|c|c|c|c|c|c|}
\hline País & $\begin{array}{l}\text { Categoría } 1 \\
\text { (en la calle) }\end{array}$ & $\begin{array}{c}\text { Categoría } 2 \\
\text { (en alojamiento } \\
\text { de emergencia } \\
\text { como albergues } \\
\text { nocturnos) }\end{array}$ & $\begin{array}{c}\text { Categoría } 3 \\
\text { (en alojamientos } \\
\text { temporales para } \\
\text { personas sin } \\
\text { hogar) }\end{array}$ & $\begin{array}{c}\text { Categoría } 4 \\
\text { (en instituciones } \\
\text { penales o } \\
\text { sanitaria) }\end{array}$ & Año de referencia \\
\hline \multirow{3}{*}{ Austria } & \multicolumn{2}{|c|}{13.926} & 8.688 & & 2017 \\
\hline & & & 3.284 & & 2018 \\
\hline & & & & 9.490 & 2019 \\
\hline \multirow{3}{*}{ Bélgica } & 511 & 1.452 & & \multirow{3}{*}{ ND } & 2017 \\
\hline & ND & 764 & & & 2014 \\
\hline & & & 4.694 & & $2014-15$ \\
\hline Alemania & 52.000 & $N / D$ & $\mathrm{~N} / \mathrm{D}$ & $\mathrm{N} / \mathrm{D}$ & 2016 \\
\hline Dinamarca & 648 & 305 & 4.238 & 217 & 2017 \\
\hline Grecia & 691 & $N / D$ & 954 & $N / D$ & 2018 \\
\hline España & 3.149 & $9.915^{4}$ & 4.879 & $\mathrm{~N} / \mathrm{D}$ & 2012 \\
\hline Finlandia & & & 610 & 713 & 2018 \\
\hline \multirow{3}{*}{ Francia } & 8.700 & 9.000 & & \multirow{3}{*}{$\mathrm{N} / \mathrm{D}$} & 2019 \\
\hline & & & 25.000 & & 2013 \\
\hline & & & 132.000 & & 2015 \\
\hline \multirow{3}{*}{ Irlanda } & 156 & \multirow{2}{*}{$\mathrm{N} / \mathrm{D}$} & & \multirow{3}{*}{$N / D$} & 2018 \\
\hline & 127 & & & & 2016 \\
\hline & & & 10.305 & & 2019 \\
\hline Italia & 28.773 & 25.939 & 23.367 & $\mathrm{~N} / \mathrm{D}$ & 2014 \\
\hline \multirow{5}{*}{ Luxemburgo } & 30 & ND & & & 2006 \\
\hline & ND & 773 & & & 2018-19 \\
\hline & ND & 93 & & & 2018 \\
\hline & & & 3.723 & & 2016-18 \\
\hline & & & 337 & 220 & 2014 \\
\hline \multirow{2}{*}{ Países Bajos } & \multicolumn{2}{|c|}{30.500} & & & 2016 \\
\hline & & & 70.000 & 8.777 & 2017 \\
\hline Portugal & 1.443 & 210 & 2.218 & 810 & 2018 \\
\hline Suecia & 1.647 & 1.229 & 19.530 & 4.899 & 2017 \\
\hline \multirow[t]{3}{*}{ Reino Unido } & 5.711 & & & \multirow{2}{*}{$\mathrm{N} / \mathrm{D}$} & $\begin{array}{l}\text { 2018, excepto } \\
\text { para Escocia } \\
(2014)\end{array}$ \\
\hline & $N / D$ & 42.200 & & & 2016 \\
\hline & & & 97.469 & & 2018-19 \\
\hline TOTAL & & & 411.296 & 25.126 & \\
\hline
\end{tabular}

Fuente: Elaboración propia a partir de Baptista y Marlier (2019).

estimarse en torno a 240.000 personas viviendo en la calle o en albergues nocturnos, la mayor parte de las cuales (el $77 \%$ ) estarían concentradas en Italia, Alemania, Reino Unido y Países Bajos. En torno al $5,5 \%$ estarían en España ${ }^{5}$. De las cuatro categorías analizadas, la mayor parte de las personas sin hogar estarían en alojamientos temporales para personas sin hogar (411.00o). Más de la mitad de los países no dispone de información sobre personas viviendo en instituciones penales o sanitarias.

4 Este dato se refiere de manera conjunta a personas sin hogar en albergues (categoría 2) o residencias (categoría 3), con lo que una parte de estos datos podrían haberse considerado en la categoría 3.

5 Como comentaremos más adelante, debe tenerse en cuenta que los datos no reflejan las consecuencias de la crisis después de 2012 y que no fueron encuestadas las personas sin hogar que no asistían a los centros para personas sin hogar.
Las estrategias de lucha contra el sinhogarismo en Europa son muy dispares, aunque cada vez más países europeos están desarrollando estrategias integrales de ámbito nacional o regional. La mayoría de los países tienen estrategias específicas para personas sin hogar a nivel nacional o a nivel regional o municipal ${ }^{6}$. Otros países incluyen medidas contra el sinhogarismo dentro de estrategias de política social

${ }^{6}$ Se trataría de los siguientes 16 países: Austria, Bélgica, Alemania, Suecia, Eslovaquia, Reino Unido, República Checa, Dinamarca, España, Finlandia, Francia, Irlanda, Italia, Luxemburgo, Países Bajos y Portugal. De todos ellos, los diez últimos tienen una estrategia específica de ámbito nacional. Grecia está en proceso de elaboración de una estrategia de ámbito nacional (Ziomas et al., 2019). 
más amplias ${ }^{7}$ y diez más ${ }^{8}$, la mayoría de ellos países del Este, no tienen un enfoque específico contra el sinhogarismo aunque incluyen algunas medidas en políticas sociales relacionadas con vivienda, servicios sociales, pobreza e inclusión social o políticas de garantía de rentas.

Entre los países con estrategias de ámbito nacional existen algunos elementos comunes, de los que destacamos dos. En primer lugar, la participación de un amplio rango de grupos de interés públicos y privados en el diseño de las estrategias. Tal sería el caso, por ejemplo, de la República Checa en su estrategia aprobada en 2013 y en vigor hasta el 2020 (Sirovátka et al., 2019) o de la Estrategia Nacional Integral para Personas sin Hogar 2015-2020 en España. En segundo lugar, el creciente protagonismo aunque aún minoritario, del enfoque Housing First como eje vertebrador del apoyo a las personas sin hogar. Destacarían países como la República Checa o España. También Italia, a través de la adopción en diciembre de 2015 de las Directrices para Afrontar la Marginación Adulta Severa Jessoula et al., 2019), Luxemburgo (Estrategia Nacional contra el Sinhogarismo y la Exclusión Residencial 2013-2020", aprobada en 2013) (Urbé, 2019), los Países Bajos mediante el Programa de Estímulo del Housing First (Oostveen, 2019), incluso, aunque de manera menos decidida, Portugal a través de la Estrategia Portuguesa para la Integración de Personas sin Hogar 2017-2023 aprobada en 2017 (Perista, 2019). La información sobre la financiación de las estrategias contra el sinhogarismo es igualmente muy heterogénea. Encontramos países como Bélgica, Estonia, España, Montenegro o Macedonia del Norte, donde no es posible determinar la financiación destinada a sus estrategias. No obstante, en la mayoría de los países existen evidencias de que la financiación resulta insuficiente e inadecuada y, en muchos otros (trece en total), existen considerables diferencias en la capacidad de financiación entre las diferentes regiones y municipios dentro de cada país.

En lo relativo al tipo de servicios, en la mayoría de los países prevalece un "modelo de escalera" frente a un modelo de Housing Led/Housing First que todavía resulta minoritario ${ }^{9}$. No obstante, en cada vez más países con un modelo de escalera se están introduciendo iniciativas de Housing First a escala local (caso de Suecia, Rumanía o Portugal, entre otros) o tendencias de cambio más profundas en las que este modelo va ganando peso en el conjunto de

7 Bulgaria, Croacia, Polonia, Rumanía, Albania, Macedonia del Norte y Serbia.

${ }^{8}$ Chipre, Estonia, Hungría, Letonia, Lituania, Malta, Eslovenia, Bosnia y Herzegovina, Montenegro y Kosovo.

9 Se define el "modelo de escalera" como un proceso de inclusión social progresivo que culmina en el acceso a la vivienda renunciando a iniciar el proceso justo por el acceso a la vivienda, como se produce en el modelo de Housing First. En los siguientes países predomina un modelo de escalera: Bosnia y Herzegovina, Bulgaria , República Checa, Chipre, Estonia, Grecia, Croacia, Lituania, Letonia, Montenegro, Macedonia del Norte, Malta, Polonia, Serbia y Turquía; el modelo Housing First predomina en Albania, Dinamarca, Finlandia, Países Bajos y Reino Unido. los servicios orientados a personas sin hogar (caso de Francia, Bélgica, Italia, Austria, Irlanda o España). En general, más de veinte países señalaron tener algún programa, proyecto o estrategia basada en el modelo de Housing First (Baptista y Marlier, 2019) pero, como señalábamos antes, aún dista mucho de ser un servicio ampliamente extendido dentro de los servicios orientados a las personas sin hogar en Europa.

Los servicios de prevención del sinhogarismo son muy variados en la UE. Generalmente están orientados a potenciales personas en situación de sinhogarismo y abarcan servicios como, entre otros, mecanismos de detección de desahucios (publicación de las órdenes de desahucio de los tribunales de justicia, procedimientos de alerta ante desahucios implicando a diferentes niveles administrativos), mediación de conflictos con los arrendadores y asesoramiento sobre financiación de deudas hipotecarias, apoyo económico para los impagos de las rentas de alquiler o acceso prioritario a realojos en viviendas. Por lo general, los servicios preventivos en Europa no están adecuadamente focalizados ni diseñados para la detección precoz de potenciales situaciones de sinhogarismo (Baptista y Marlier, 2019).

Prácticamente en la totalidad de los países europeos la provisión de servicios recae casi exclusivamente en el ámbito local, con un papel muy relevante de las ONG, organizaciones de la sociedad civil y autoridades locales. La responsabilidad de la provisión de servicios pivota sobre la administración local bien de manera directa o contratada con las ONG, las cuales rara vez desarrollan otras actividades que no sean de provisión directa de servicios (por ejemplo, no realizan el seguimiento o la evaluación). En algunos países, los municipios no solo proveen servicios sino que también desempeñan una función relevante en la planificación, coordinación, regulación, seguimiento y financiación de los servicios10. En la mayoría de los países, la Administración central también es responsable de la financiación y en apenas una docena de ellos juega un papel relevante en la planificación y regulación11 y en el seguimiento y evaluación de los servicios para personas sin hogar. Solo en unos pocos países (por ejemplo, Austria, Bélgica, República Checa, España o Francia) las autoridades regionales también desempeñan un importante papel en la planificación, coordinación y regulación de la provisión de servicios $y$, en menor medida, en la financiación de tales servicios.

${ }^{10}$ Por ejemplo, en Dinamarca, Alemania, Italia, Letonia, Lituania, Países Bajos, Portugal, Rumanía y Suecia.

${ }^{11}$ Esto se produce en Bélgica, Chipre, República Checa, Dinamarca, Estonia, Grecia, Finlandia, Italia, Luxemburgo, Eslovenia, Eslovaquia y Kosovo. 


\section{El sinhogarismo en España: naturaleza, alcance y principales políticas}

\subsection{Naturaleza y alcance del sinhogarismo en España: apuntes básicos}

La Estrategia Nacional Integral para Personas sin Hogar 2015-2020 ${ }^{12}$ (en adelante ENIPSH 2015-2020) constituye el primer instrumento de ámbito estatal para abordar la situación de las personas sin hogar. La definición de sinhogarismo utilizada en la ENIPSH 2015-2020 está basada en la categorización ETHOS e incluye las categorías 1 a 4 de ETHOS light. Esta estrategia no considera los criterios de ETHOS relacionados con la exclusión residencial asociada a situaciones de vivienda insegura e inadecuada. En España estas situaciones suelen abordarse dentro de políticas más amplias relacionadas con lucha contra la exclusión social y la exclusión de la vivienda. Aunque el objetivo de este artículo es centrarse en el sinhogarismo, también haremos alguna mención a las políticas relacionadas con la exclusión residencial en la medida en la que pueden tener influencia en la trayectoria de las personas hacia situaciones de sinhogarismo y porque la tendencia cada vez más predominante es hacia lograr una vivienda en la comunidad para las personas sin techo.

Los últimos datos disponibles a nivel nacional sobre personas sin hogar son muy antiguos y proceden de la Encuesta de Personas sin Hogar del año 2012 elaborada por el Instituto Nacional de Estadística (INE) ${ }^{13}$. España, junto con Rumanía (2008), BosniaHerzegovina (2013) o Serbia (2011), se encuentra entre los países europeos con datos oficiales de personas sin hogar más antiguos de entre los 35 países europeos analizados (Baptista y Marlier, 2019).

En esta encuesta en 2012 se contabilizaron 22,938 personas sin hogar atendidas en centros asistenciales ${ }^{14}$, lo que supuso un aumento del 4,7 $\%$ con respecto a la anterior encuesta realizada en $2005^{15}$, que las cifraba en 21.900 personas.

Estos datos necesitan ser actualizados y además tienen dos limitaciones importantes: no reflejan las consecuencias de la crisis después de 2012 y no fueron entrevistadas las personas sin hogar que no asistían a los centros para personas sin hogar. Por ello, como mencionaremos después, algunas ciudades están realizando sus propios recuentos

${ }^{12}$ Disponible en 〈http://bit.ly/2UBndsC .

${ }^{13}$ Disponible en 〈http://bit.ly/2UBnsUy〉.

${ }^{14}$ La Encuesta del INE es anterior a la ENIPSH 2015-2020 y no fue diseñada siguiendo las categorías de ETHOS light. No obstante, del análisis de los datos podemos señalar que las 22.938 personas sin hogar estimadas en la encuesta se distribuirían de la siguiente manera atendiendo a las categorías de ETHOS light: categoría 1 (6.362); categoría 2 (9.915); categoría 3 (4.879); categoría 6 (1.738); sin clasificar (44). Advertir que el dato reflejado en la categoría 2 se refiere de manera conjunta a personas sin hogar en albergues o residencias, con lo que también podría haberse considerado este dato en la categoría 3.

${ }^{15}$ Nota de Prensa: 〈http://www.ine.es/prensa/np398.pdf〉. nocturnos de personas que no están pernoctando en centros asistenciales. Considerando los datos de la encuesta de 2012 y extrapolando los recuentos del País Vasco y de ciudades como Madrid, Zaragoza y Barcelona ${ }^{16}$ al conjunto del Estado, en la ENIPSH 2015-2020 se estimó que el número total de personas sin alojamiento ni vivienda en España podría situarse en torno a las 33.000 personas en 2014 (ENIPSH 2015-2020; Rodríguez Cabrero y Marbán, 2016). ONG como Cáritas estiman que en la actualidad están acompañando y atendiendo aproximadamente a 40.000 personas sin hogar en España.

Posteriormente a la aprobación a la ENIPSH 20152020, regiones como el País Vasco y algunas ciudades como Madrid, Barcelona, Zaragoza o Las Palmas de Gran Canaria ${ }^{17}$ han elaborado sus propios planes y estrategias para personas sin hogar, todos ellos inscritos en el marco establecido en la ENIPSH 2015-2020. Las ciudades de Madrid, Barcelona, Zaragoza y las capitales del País Vasco también han actualizado los recuentos de personas sin hogar y cada vez más ciudades (como Alicante, Mallorca o Las Palmas) y regiones (Galicia ${ }^{18}$ ) están elaborando nuevos recuentos. En general, en todos los recuentos aumenta el número de personas sin hogar (un $25 \%$ en Madrid entre 2016 y 2018; un 3,3 \% en Zaragoza entre 2012 y 2016; un 1,6 \% en Barcelona entre 2013 y 2017 o un 9,4 \% en el País Vasco entre 2014 y 2016). En todas, salvo en Madrid, disminuyen las personas durmiendo en la calle (un $34 \%$ en Zaragoza, un $30 \%$ en Barcelona y un $15 \%$ en País Vasco) y aumentan las personas sin hogar alojadas en centros (un $11 \%$ en Madrid, un 29 \% en Zaragoza, un 33 \% en Barcelona y un $15 \%$ en el País Vasco $)^{19}$.

${ }^{16}$ En la ciudad de Madrid, en 2014 se localizaron 1.905 personas sin hogar (Muñoz et al., 2014); 455 en Zaragoza en el año 2012 (Cabrera, 2012); 2.933 personas en Barcelona en el año 2013 (Sales, 2013); 1.836 en las tres capitales del País Vasco en 2014 (Fundación Eguía-Careaga, 2015).

${ }^{17}$ Estrategia Vasca para Personas sin Hogar, 2018-2021, 〈http:// bit.ly/2PpBffY>; Estrategia Municipal Para la Prevención y Atención al Sinhogarismo, 2015-2020, 〈http://bit.ly/2Dwonge〉. La región de Madrid también ha elaborado un Plan de Inclusión de Personas Sin Hogar 2016-2021, 〈http://bit.ly/2GtATB5〉; Plan de lucha contra el sinhogarismo de Barcelona 2016-2020, 〈http://bit.ly/2GuGneF〉; Propuesta de Plan Integral para las personas sin hogar en Zaragoza (2018), 〈http://bit.ly/2vjlolk〉; Plan Estratégico de Atención Integral a Personas sin Hogar en Las Palmas de Gran Canaria 2017-2021, 〈http://bit.ly/2Gy2Nfj〉.

${ }^{18}$ Actualmente también se está elaborando el Plan de Personas sin Hogar de Galicia.

${ }^{19}$ En la ciudad de Madrid, en 2018 las personas sin hogar (2,583: 1933 personas en centros y pisos y 650 personas viviendo en la calle) aumentaron un $25 \%$ con respecto a $2016(2,059)$ (IX Recuento de Personas Sin Hogar en Madrid 2018, 〈https://faciam.org/tag/ recuentos/ >). En Zaragoza en 2016 fueron detectadas 470 personas sin hogar (347 personas en centros y 123 personas viviendo en la calle) ( 455 en 2012) (Propuesta de Plan Integral para las personas sin hogar en Zaragoza,2018). En Barcelona, en 2017 se contaron 2,980 personas sin hogar (1.954 alojadas en equipamientos y 1.026 personas durmiendo en la calle -2,933 en 2013-) (Sales, 2013), 〈http:// bit.ly/2W1 (u1b)). En el País Vasco en el periodo 2014 -2016 las personas sin hogar aumentaron un 9,42 \% (1,836 en 2014; 2,009 en 2016: 1735 en centros y 274 en la calle) (Estrategia Vasca para Personas Sin Hogar, 2018-2021). En Galicia, en 2017 se contaron 340 personas sin hogar (211 en centros y 129 en la calle personas sin hogar), 〈http:// bit.ly/2Uv1owp〉; en Las Palmas de Gran Canaria, en 2016 se contaron 361 personas ( 280 personas en centros y 81 en la calle) <http://bit. ly/2Gy2Nfj>; en Alicante, en 2017195 personas durmiendo en la calle, 
Este aumento de personas sin hogar alojadas en centros puede estar relacionado con el incremento en el número de centros de atención a personas sin hogar en el conjunto del Estado. En la última encuesta realizada por el INE en el año 2016 sobre centros y servicios de atención a personas sin hogar ${ }^{20}$, se observa que la oferta de plazas para atender en centros a las personas sin hogar creció un $14,62 \%$ en 2016 respecto a 2014 (pasando de 16.684 en 2014 a 19.124 en 2016). La media diaria de personas que se alojaron en centros de atención a personas sin hogar creció un 20,46\% en 2016 respecto a 2014 (de 13.645 a 16.437 plazas ocupadas en 2016). La ocupación media alcanzó el 85,9\%, cifra superior a la observada en 2014 , que fue del $81,8 \%$. En esta encuesta se recoge por primera vez el programa de Housing First, con un aumento de las viviendas disponibles para este programa que pasaron de 132 en junio de 2016 (ocupadas por 233 personas) a 171 en diciembre (ocupadas por 271 personas).

Como señalábamos más arriba, la ENIPSH 20152020 no incluye a personas en situación de exclusión residencial asociada a vivienda insegura e inadecuada. Para conocer su alcance es necesario recurrir a estudios como el de la Fundación Foessa (2018 a y b), cuya encuesta estima que casi 800.000 hogares sufren situaciones de inseguridad en la vivienda (cuatro de cada cien hogares), 1.300.000 hogares sufren inadecuación en la vivienda (siete de cada cien hogares) y 150.000 hogares sufren ambas situaciones a la vez. La inadecuación de la vivienda en España también es plasmada por Feantsa y Abbé Pierre Foundation (2019) en su informe sobre la exclusión de la vivienda en Europa de 2019, al señalar que en 2017 la proporción de hogares sobrecargados por los costes de la vivienda fue del $36,5 \%$ entre la población en situación de pobreza y del 9,8 \% entre la población total. La proporción de hogares con atrasos en el pago de alquileres o hipotecas es la séptima más alta de Europa (10,5\% entre los hogares pobres y 3,8 \% entre la población total). El 19,4\% de los hogares pobres tienen dificultades económicas para mantener una temperatura adecuada en sus hogares, frente al $8 \%$ de la población total.

En cuanto a la evolución de las personas sin hogar, aunque las fuentes manejadas no son del todo comparables, los datos señalados arriba apuntan hacia un aumento de las personas sin alojamiento ni vivienda desde hace más de una década. Varias estrategias regionales de personas sin hogar coinciden en señalar que el aumento del sinhogarismo se debe a carencias económicas asociadas a la crisis y a carencias relacionales o psicosociales. Según el INE, en 2012 el $45 \%$ se quedó sin hogar porque perdió el trabajo; la segunda razón fue no haber podido pagar más su alojamiento (26\%) y la tercera causa fue la separación de su cónyuge 0 pareja (20,9\%).

〈http://bit.ly/2Lo03zN 〉; en Mallorca, 209 personas durmiendo en la calle en 2017, 〈http://bit.ly/2KXSCp)>.

${ }^{20}$ Nota de prensa: 〈http://bit.ly/2UDgKos〉.

\subsection{Principales estrategias y políticas dirigidas a luchar contra el sinhogarismo en España}

El análisis de las estrategias y políticas dirigidas a luchar contra el sinhogarismo debe hacerse en el marco de la crisis económica y financiera y su impacto social en España durante el período 2012-2018.

Previamente a la aprobación en 2015 de la ENIPSH 2015-2020, las políticas de lucha contra el sinhogarismo tenían como marco institucional de orientación y regulación el Plan Nacional de Acción para la Inclusión Social 2013-2016. Este plan contemplaba diferentes acciones para afrontar problemas asociados a la vivienda incluyendo el sinhogarismo. También el Plan Integral de Apoyo a la Familia 2015-2017 incluye el desarrollo de medidas de apoyo para el acceso a una vivienda digna adaptada a las necesidades de cada familia, potenciando la erradicación de la vivienda deficiente y protegiendo a las personas hipotecadas con responsabilidades familiares.

Este doble marco institucional servía de referencia a las Comunidades Autónomas y Ayuntamientos que son los que tienen competencias exclusivas en políticas de vivienda y de lucha contra la exclusión social.

El impacto de la crisis económica y financiera movilizó a la sociedad civil en la lucha contra el sinhogarismo y la exclusión de la vivienda. Entidades tradicionalmente muy activas como RAIS, Cáritas Española o Faciam, y otras creadas en los años de mayor impacto de la crisis como la Plataforma de Afectados por la Hipoteca (PAH) favorecieron la aprobación de programas y medidas para hacer frente al mayor riesgo de exclusión de la vivienda como el Fondo de Vivienda Social creado en 2013, la Ley $1 / 2013$ de medidas para reforzar la protección a los deudores hipotecarios, reestructuración de deuda y alquiler social ${ }^{21}$, o el Real Decreto 897/2017 de 6 de octubre por el que se regula la figura del consumidor vulnerable, el bono social y otras medidas de protección para los consumidores domésticos de energía eléctrica con el fin de paliar las privaciones materiales y los costes asociados a la vivienda (Arriba y Rodríguez Cabrero, 2018).

Todas estas políticas fueron respuestas dirigidas a aliviar situaciones de exclusión social que exigían una respuesta urgente, sobre todo las relacionadas con la pérdida de la vivienda que podrían devenir en sinhogarismo. Las limitaciones de estas políticas, las recomendaciones del Parlamento Europeo y la demanda del Tercer Sector de Acción Social (TSAS)

\footnotetext{
${ }^{21}$ Algunas medidas de esta ley se ampliaron a cuatro años en 2015 con el Real Decreto-ley 1/2015, de 27 de febrero («http://bit. ly/2(DB1al)). Posteriormente, el Real Decreto-ley 5/2017 modificó la Ley 1/2013 de medidas para reforzar la protección a los deudores hipotecarios, reestructuración de deuda y alquiler social. Este Real Decreto, por ejemplo, amplía el plazo hasta marzo de 2020 de la moratoria para los desalojos a raíz de las ejecuciones hipotecarias.
} 
que trabaja contra la exclusión social, también favorecieron el desarrollo de dos estrategias que comentamos a continuación: una general, a través de planes nacionales de vivienda, y otra dirigida expresamente a la lucha contra el sinhogarismo, la mencionada ENIPSH 2015-2020.

- La estrategia general de acceso a la vivienda en España se concreta en el Plan Nacional de Vivienda 2018-2021 que, en cierto modo, actualiza el Plan de Vivienda 2013-2016, cuya evaluación no ha visto la luz. El nuevo plan contempla planes de apoyo a la vivienda de alquiler, renovación de viviendas y la rehabilitación urbana, y tiene por objeto facilitar el acceso a la vivienda de los grupos más vulnerables. Ciertamente, estos objetivos ya se contemplaban en el plan del período 2013-2016, pero en la práctica no habían sido aplicados.

- La Estrategia Nacional Integral para Personas sin Hogar 2015-2020 fue aprobada en noviembre de 2015 a raíz de una iniciativa parlamentaria aprobada en abril de 2014 en la que el TSAS desempeñó un papel importante. La estrategia se planteó dentro del marco del Plan Nacional de Acción para la Inclusión Social 2013-2016 y en colaboración con las comunidades autónomas, administraciones locales y el TSAS, integrando las perspectivas de política social y de vivienda así como de salud, trabajo o educación con el fin de dar cumplimiento a las resoluciones del Parlamento Europeo.

En la práctica, el desarrollo de esta estrategia ha estado paralizado debido a una falta de compromiso institucional y financiero. El 21 de septiembre de 2017, el Congreso de los Diputados aprobó una nueva proposición no de ley para impulsar el desarrollo de la estrategia aprobada en 2015. La propuesta consistió en aprobar un Plan de Acción Integral para las Personas sin Hogar de acuerdo con las comunidades autónomas, los gobiernos locales y el TSAS. Esta propuesta no ha sido llevada aún a la práctica ${ }^{22}$.El enfoque de la estrategia es "la aplicación prioritaria de enfoques orientados a la vivienda Housing Led”. Esta estrategia se concreta en tres principios: Housing First, frente a la política tradicional que priorizaba los albergues municipales; atención integral y centrada en las personas sin hogar comprendiendo el empleo, la educación y la sanidad; y diversidad servicios de apoyo cuya intensidad varía entre comunidades autónomas y gobiernos locales.

La ENIPSH supone así el marco institucional mediante el que se promueve la acción conjunta del Estado central con las comunidades autónomas y el TSAS. Pero son los gobiernos regionales y locales los que en realidad asumen la competencia en el desarrollo

${ }^{22}$ Desde el propio Ministerio de Sanidad, Consumo y Bienestar Social se reconoce la necesidad de revitalizar dicha estrategia. En la fecha de realización de este artículo aún no se había realizado la evaluación intermedia prevista de la Estrategia. de la estrategia. En el caso concreto de la lucha contra el sinhogarismo, la capacidad financiera del Estado central a través del programa del o,7 $\%$ del IRPF ha sido transferida a las comunidades autónomas, que controlan el 80 \% de la financiación de este programa.

La financiación de la ENIPSH se contempla de manera genérica en el documento programático. Bajo un epígrafe general denominado "Evaluación de la financiación de la Estrategia”, se afirma que la financiación de la estrategia recae en la administración central, las comunidades autónomas y los gobiernos locales de acuerdo con el actual sistema de descentralización de las políticas sociales. La concreción de dicha financiación y su reparto se hará en base a los planes de atención integral a personas sin hogar. Al no haberse desarrollado el primer plan nacional integral se desconoce el volumen de la inversión prevista y su reparto. Se afirma que: "cuando se diseñen los Planes de Atención Integral en cada territorio, se podrá concebir la escala de inversión necesaria en cada una de las comunidades autónomas y administraciones locales". En consecuencia, el gasto público de las políticas de lucha contra el sinhogarismo no sigue aún una lógica estratégica, sino la tradicional senda de gasto de las distintas administraciones implicadas.

Como ya se ha comentado, la ENIPSH 2015-2020 ha servido como marco de referencia de las estrategias regionales y municipales contra el sinhogarismo. El objetivo de estas páginas no es analizar dichas estrategias, pero, en general, tienen un denominador común como es la orientación hacia el enfoque Housing Led y la importancia de la perspectiva de género. La revisión de las estrategias y planes de acción que hemos llevado a cabo no proporciona información suficiente sobre dos temas centrales: ni sobre los indicadores de seguimiento y evaluación ni sobre la inversión financiera prevista para la aplicación del modelo de Housing First. En cualquier caso, los nuevos planes municipales y las nuevas estrategias regionales comparten el abandono progresivo de un enfoque asistencialista en favor de una intervención integral de apoyo a las personas en situación de exclusión habitacional (Federación de asociaciones de centros para la integración y ayuda de marginados, 2015; Duque, 2014; SIIS, 2015).

La estrategia nacional incluye un conjunto de indicadores que deben ser desarrollados entre los diferentes niveles de gobierno y el tercer sector de acción social (TSSA). Muchos de ellos siguen el camino de la experiencia de Feantsa. Sin embargo, a falta de un plan de acción, no es posible extraer conclusiones sobre la aplicación final del sistema de indicadores. Por otra parte, las estrategias regionales y municipales informan con cierto detalle sobre sus objetivos en relación con los grupos en situación de exclusión, pero no proporcionan información sobre los indicadores finales ni sobre la financiación. 
En marzo de 2019 se ha aprobado la Estrategia Nacional de Prevención y Lucha contra la Pobreza y la Exclusión Social 2019-2023 (MSCBS, 2019). Dado que sustituye al Plan Nacional de Inclusión Social 2013-2016 como marco institucional de referencia para las políticas de lucha contra la exclusión social, cabe esperar que tenga cierta incidencia en el marco general de las políticas de vivienda y sinhogarismo, incluida la ENIPSH 2015-2020, en los próximos años. En la nueva estrategia de lucha contra la pobreza, se incluye a las personas sin hogar como grupo particularmente vulnerable y con un carácter prioritario en las actuaciones en materia de vivienda. También se propone articular una política de prevención y lucha contra el sinhogarismo, con el fin de que las personas $\sin$ hogar restauren su proyecto de vida y se reincorporen a la sociedad. Sin embargo, la falta de una financiación concreta para ser desarrollada y aspectos como la ausencia de vinculaciones concretas entre las medidas de garantía de ingresos y las de garantía de acceso a la vivienda (Sanzo, 2019) exigen cautela sobre su impacto en el sinhogarismo.

\section{Efectividad, innovaciones y principales limitaciones y prioridades de los servicios de apoyo a las personas sin hogar en España y la Unión Europea}

En el ámbito europeo existen amplias diferencias sobre la evidencia disponible en cuanto a la implementación y seguimiento de las estrategias de apoyo a las personas sin hogar. En países como Dinamarca, Francia, Finlandia o Irlanda existen mecanismos robustos de evaluación. Otros, como la República Checa, Alemania, Portugal, Suecia o Reino Unido los están poniendo en marcha. No obstante, en la mayoría de los países falta sistematicidad en los procesos de seguimiento y evaluación de la efectividad de los servicios para personas sin hogar. Los principales obstáculos suelen ser el nivel de compromiso institucional y político, el grado de cooperación y coordinación entre los departamentos y administraciones implicadas y la insuficiencia de las fuentes de información disponibles y de mecanismos adecuados de financiación (Baptista y Marlier, 2019).

Como ejemplos de buenas prácticas de evaluación y seguimiento se pueden destacar a Dinamarca o Finlandia. En Dinamarca, su estrategia ha sido evaluada por dos organismos autónomos externos y cada dos años se realiza una cartografía de las personas sin hogar (Oostveen, 2019). En Finlandia, el seguimiento de la efectividad de las estrategias se efectúa a partir de las estadísticas oficiales realizadas tanto a nivel nacional como local y con cada cambio de gobierno (generalmente cada cuatro años) se realiza un exhaustivo estudio para evaluar los resultados de las políticas públicas (Kangas y Kalliomaa-Puha, 2019). En la República Checa (Sirovátka et al., 2019) y Francia (Legros, 2019) se han establecido grupos de trabajo interministeriales con el fin de supervisar y evaluar los progresos realizados en la implementación de sus estrategias y desde 2014 ya se han realizado diversos informes al respecto.

En el ámbito de la innovación existe una amplia diversidad de iniciativas que han ido en aumento en los últimos cinco años. La más destacada es la introducción o el desarrollo de servicios de Housing First. Baptista y Marlier (2019) han identificado otras iniciativas de innovación como las siguientes: equipos de futbol en Bélgica para la integración social de personas sin hogar, o lavanderías y duchas móviles para personas sin hogar en Atenas; introducción de cambios legales para garantizar unos estándares mínimos de calidad en los servicios para personas $\sin$ hogar; medidas orientadas a intensificar la construcción de pequeños apartamentos para personas $\sin$ hogar (por ejemplo, 1,5 millones de viviendas entre 2018 y 2021 en Alemania) o viviendas "diminutas" de más rápida construcción y menor coste para personas solas sin hogar en el Reino Unido; finalmente, medidas para mejorar la efectividad de programas y servicios para personas sin hogar como el modelo danés de Inversión SocioEconómica $(S \varnothing \mathrm{M})^{23}$, o el proyecto finlandés AUNE de 2016-2019 (Kangas y Kalliomaa-Puha, 2019), que permite identificar grupos de riesgo de sinhogarismo y cuyo objetivo es construir 2.500 alojamientos para personas sin hogar y proveerles orientación y servicios de prevención individualizados.

A pesar de estas innovaciones las estrategias de lucha contra el sinhogarismo siguen presentando importantes limitaciones. La limitación más destacada por la gran mayoría de países es el restrictivo diseño de las políticas públicas de vivienda y el propio mercado de la vivienda que llevan asociados crecientes precios de la vivienda y del alquiler, stock limitado de vivienda social asequible, escasa oferta pública de vivienda en propiedad y en alquiler, y desajustes entre la oferta y demanda de vivienda fundamentalmente debidos a la liberalización del mercado del alquiler y la baja inversión pública en vivienda social.

También destacan la falta de estrategias integradas entre los distintos niveles de la administración (local, regional y nacional) lo que no solo afecta a la eficacia de dichas estrategias sino también a la equidad territorial en el alcance y calidad de la provisión de servicios para personas sin hogar. A ello se unen otros factores organizativos que han sido señalados por expertos de países como Austria, República Checa, Grecia, España, Finlandia, Francia, Polonia o Reino Unido, como la limitada información estadística y de bases de datos o recursos humanos insuficientes o con baja cualificación. Finalmente, la baja inversión en prevención, unida

${ }^{23}$ Este modelo se lanzó en enero de 2018. Sirve para calcular el coste y beneficio de las intervenciones y su impacto presupuestario. Se utiliza como banco de datos sobre los efectos sociales y económicos de las intervenciones para los diferentes grupos de personas sin hogar a los que se orienta y puede usarse para la toma de decisiones por parte de las autoridades públicas (más detalles en Kvist, 2019). 
al empeoramiento de causas estructurales del sinhogarismo como la pobreza, el desempleo, el bajo nivel de protección social y la creciente inestabilidad y precariedad laboral, son factores que siguen ocluyendo la transición de las personas sin hogar hacia su inclusión residencial.

Avanzar en dicha transición pasa por mejorar la provisión de servicios para las personas sin hogar. En el trabajo de Baptista y Marlier (2019) que venimos mencionando se han destacado una serie de prioridades de intervención en las que coinciden la mayor parte de los treinta y cinco países europeos analizados.

En políticas de vivienda se considera inaplazable ampliar el stock de viviendas públicas y sociales, construyendo más viviendas asequibles para personas de bajos ingresos y restaurando viviendas en condiciones precarias, actuando sobre el aumento de los costes de la vivienda, así como regulando la oferta de viviendas de alquiler con medidas más estrictas de control de alquileres y regulación del sector de la vivienda privada, especialmente en lo que respecta a los derechos de los inquilinos.

En segundo lugar, mejorar la gobernanza en política de vivienda pública y en la coordinación institucional, avanzando en la cooperación entre las partes interesadas (municipios, regiones, administración central, asociaciones de vivienda, organizaciones de atención y ONG) e introduciendo unos estándares adecuados para el apoyo a las personas sin hogar.

Para permitir una mayor comprensión del fenómeno y el seguimiento y evaluación de la situación de las personas sin hogar, resultaría necesario mejorar el sistema de recopilación de información sobre personas sin hogar asegurando estadísticas uniformes a nivel nacional. Igualmente debería ser prioritario el desarrollo de una política preventiva más estructural que no se centre solo en los grupos más visibles de personas sin hogar, aumentar la protección legislativa contra los desalojos o introducir coordinadores personales/gestores de caso para ayudar a navegar por el sistema de protección y poder coordinar las medidas orientadas a las personas sin hogar.

En el caso de España, el desarrollo del sinhogarismo durante los últimos cinco años, sobre todo desde la aprobación de la ENIPSH, permite identificar algunas debilidades y prioridades fundamentales para el desarrollo de políticas omnicomprensivas.

Entre las principales limitaciones destacaríamos las siguientes:

- Escasa relevancia de los programas de prevención del sinhogarismo. Según la encuesta realizada por el INE en el año 2016 sobre centros y servicios de atención a personas sin hogar ${ }^{24}$, el

${ }^{24}$ Nota de prensa: 〈http://bit.ly/2UDgKos〉.
$36,9 \%$ de los centros declaró que su actividad principal era proporcionar alojamiento en pisos o apartamentos, el $24,8 \%$ se consideró como centro de acogida, el 9,5\% como centro de día/taller ocupacional, el 9,3\% indicó que era albergue/centro de acogida nocturno, el 8,6 $\%$ comedor social, el 4,2 \% se consideró como residencia y el $6,5 \%$ restante declaró otro tipo de actividad distinta de las anteriores. Las prestaciones más frecuentes que ofrecieron los centros fueron información y acogida (83,3 \%), alojamiento (75,7\%), orientación y/o derivación (74,9\%), atención y acompañamiento social (73,4 \%), aseo personal $(65,6 \%)$ y restauración $(65,2 \%)$. Esta tipología de servicios varía entre comunidades autónomas y entre municipios. Sin embargo, los servicios de prevención son prácticamente inexistentes.

Se puede afirmar que la oferta de servicios se encuentra en una situación de transición. Por una parte, el servicio de albergues está en crisis o solo sirve para situaciones de emergencia; es un recurso de acción inmediata y de baja intensidad pero no es un recurso con impacto a medio plazo para erradicar el sinhogarismo. Por el contrario, la oferta de servicios de acceso a la vivienda bajo la metodología de Housing First se está abriendo paso en diferentes comunidades autónomas (entre ellas, Asturias, Madrid, Cataluña, País Vasco y Andalucía) como vía de inclusión social a través de la vivienda acompañada de apoyos para el acceso al empleo. No existen como tales programas de prevención del sinhogarismo con una dimensión local o regional relevante. En general, según Rais ${ }^{25}$ se constata que o no existen o no se publican los resultados de los programas por parte de los ayuntamientos ni de las ONG. La mayoría de los informes se refieren a ratios de cobertura asistencial, sin que la prevención forme parte de la intervención social y pública. Por otra parte, la presión de la demanda conlleva dar respuesta inmediata sin capacidad suficiente para emprender programas preventivos.

- Existe también una débil coordinación entre los diferentes niveles de la Administración y baja eficacia de los servicios existentes en la prestación de un apoyo integral y flexible en función de las necesidades de las personas y en el acceso a soluciones de alojamiento permanente. La Administración o Estado central define las grandes líneas de la estrategia nacional y contribuye a la financiación de algunos programas específicos. Dado que la vivienda es competencia autonómica y local, la planificación de los programas de lucha contra el sinhogarismo tiene lugar en un doble plano, no siempre complementario: a nivel regional y a nivel local. Los planes y estrategias regionales marcan las grandes líneas de acción

${ }^{25}$ Consulta realizada el día 29 de abril de 2019 a José Manuel Caballol, director general de la Fundación Rais («https://raisfundacion. org ). 
y contribuyen a financiar una parte de los programas contra el sinhogarismo o de acceso a la vivienda en general a través de los planes regionales o autonómicos de vivienda. Por su parte, las corporaciones locales diseñan la planificación de las políticas, financian una parte importante de los programas y gestionan las prestaciones en colaboración con el sector privado no lucrativo. Este marco competencial requiere de una adecuada coordinación entre los servicios, políticas y prestaciones llevadas a cabo entre las administraciones territoriales y dicha coordinación, por lo general, sigue siendo muy débil entre los diferentes niveles de la Administración.

- Tampoco existe información adecuada sobre la efectividad de los servicios de apoyo a las personas sin hogar. Ningún albergue publica los datos de efectividad de las acciones de inclusión social de sus usuarios. En general, y de acuerdo con la muy limitada información disponible, la intervención de los servicios suele estar fragmentada según las diversas prestaciones. No existe una coordinación efectiva entre prestaciones y servicios. Las soluciones que pueden garantizar un alojamiento permanente solo benefician a una minoría de las personas sin hogar, escasamente al $10 \%$ de este colectivo.

En general, la efectividad de las políticas y programas contra el sinhogarismo choca con una serie de factores sistémicos relacionados con una insuficiente oferta de empleo personalizado o con apoyo, y de viviendas adecuadas y accesibles para las personas sin techo. Según la Fundación Rais, se estima que en torno al $77 \%$ de las personas sin techo afirman necesitar un piso para vivir $y$, sin embargo, la oferta actual de pisos, individuales o compartidos, a nivel nacional solo alcanzaría al 10 $\%$ de los actuales demandantes potenciales. Con relación al empleo, muchas personas sin techo llevan largos periodos de tiempo alejados del mercado de trabajo. En un contexto estructural de elevadas tasas de paro, el mercado de trabajo ordinario no absorbe la oferta de empleo de este colectivo. Es necesario recurrir a la demanda de empleo por parte de las empresas sociales como transición al mercado de trabajo. Este recurso $u$ otros parecidos son la alternativa más segura y efectiva para facilitar la transición al mercado ordinario de trabajo. En este contexto, la alternativa de formar a las personas sin techo antes de acceder al mercado de trabajo suele retrasar su incorporación. La experiencia parece indicar que, como principio general, el objetivo debe ser ofrecer una vivienda individual o grupal y un empleo, si es posible, al mismo tiempo; una vez integrados en una empresa social o cooperativa, se debería actuar en el ámbito de la formación y reforzamiento de habilidades laborales.

- Otro problema es la ausencia de una metodología común para hacer diagnósticos del sinhogarismo.
Los triples recuentos durante tres noches son necesarios para hacer mapas de situación fiables.

- Cabe preguntarse también si la ENIPSH 2015-2020 debe darse por cerrada e iniciarse una nueva estrategia con el nuevo ciclo político dentro de la recientemente aprobada Estrategia Nacional de Prevención y Lucha contra la Pobreza y la Exclusión Social 2019-2023. Sin un compromiso de inversión social, la actual estrategia se puede considerar totalmente estéril.

- Por otra parte, los departamentos autonómicos de vivienda y, en general, el Plan Nacional de Vivienda, son ajenos al sinhogarismo. Se centran en la financiación del acceso a la vivienda y del alquiler con contrato, pero no contemplan la realidad de las personas que viven en la calle. Donde existe una práctica de compromiso del departamento de vivienda con los programas contra el sinhogarismo (caso de Asturias) se garantiza el éxito de la acción pública.

- También asistimos al fallo de los programas de empleo ordinario para incorporar a las personas sin techo. Sin el compromiso de la economía social no es posible garantizar la integración laboral y mucho menos la transición al mercado de trabajo.

- Igualmente, se constata la ausencia de los departamentos de sanidad en la construcción de una atención sociosanitaria para un colectivo con enfermedades crónicas o muy mala salud ${ }^{26}$. Los departamentos de servicios sociales por sí solos no pueden dar respuesta a este problema.

- Junto a todo ello, existe una creciente aporofobia o delitos de odio contra el pobre que vive en la calle, que no contempla adecuadamente el Código Penal ni cuenta con una visibilidad nítida en la opinión pública.

A partir de estas limitaciones, en los próximos años deberían establecerse las siguientes prioridades en la política social contra el sinhogarismo:

- Es necesario un compromiso de inversión social a nivel estatal por parte de los ministerios de Vivienda y Sanidad y Servicios sociales.

- Sería necesario aprobar un Plan Nacional de Acción contra el Sinhogarismo con el apoyo de todos los actores institucionales y sociales y el correspondiente respaldo financiero. Desde 2015 apenas se han llevado a cabo los planes previstos en la ENIPSH 2015-2020.

- No conocemos con exactitud el volumen y características de la población sin techo, solo estimaciones. La implicación de las ONG en

${ }^{26}$ Según la última encuesta del INE sobre personas sin hogar, en 2012 el 14,2 \% de las personas sin hogar declaró tener mala o muy mala salud, el 30,7 \% alguna enfermedad crónica y el 15,2 \% alguna discapacidad reconocida. El 9,4\% de las personas mayores sin hogar sufría de alcoholismo. El 37,3\% de los jóvenes sin hogar y de personas entre 30-44 años consumían drogas (40\% entre personas de 45 a 64 años). 
el diseño de las estadísticas públicas es un paso positivo, como también lo es la aplicación de metodologías de análisis como los triples recuentos antes señalados. Es necesario actualizar los datos de la encuesta de personas sin hogar elaborada por el INE (Instituto Nacional de Estadística) (los últimos son del año 2012) incluyendo también en dicha información encuestas a las personas que no asisten a los centros para personas sin hogar.

- Potenciar la innovación en el ámbito del sinhogarismo. Cabría destacar dos tipos de innovaciones a lo largo de los últimos cinco años:

a. La primera innovación es la que se refiere al enfoque. Se ha producido un desplazamiento de la lógica asistencial basada en la oferta de albergues o residencias por la lógica del Housing First. El enfoque Housing First tiene que reforzarse, lo cual requiere que los actores sociales cooperen con mayor efectividad. En la lucha contra el sinhogarismo en la actualidad existen unas pocas ONG generalistas (Cáritas y Cruz Roja), muy pocas de tamaño medio y demasiadas ONG de pequeño tamaño. Este sector social necesita mejorar sus sinergias y cooperación para transitar hacia una política de derecho a la vivienda. En este sentido, las nuevas estrategias públicas, con el impulso e iniciativa de un sector creciente de ONG, están abandonando el llamado modelo de escalera o de responsabilidad individual, sustituyéndolo por un modelo de Housing First. Como consecuencia, las estrategias de intervención social están sustituyendo la atención en residencias y centros por la del derecho de acceso a la vivienda, si bien aún se constata una cierta resistencia al cambio por parte de los gestores de los centros y albergues.

b. La segunda innovación es el desarrollo conjunto de programas de acceso a la vivienda y la sanidad entre entidades especializadas. Un ejemplo de esta innovación es la alianza entre la principal asociación en la oferta de viviendas o pisos para personas sin hogar en España ${ }^{27}$ y la Fundación Rais. En este mismo sentido hay que destacar las experiencias de innovación en el campo de la atención sanitaria desarrolladas de manera conjunta entre ONG del sector sanitario ${ }^{28}$ y de los servicios sociales.

- La coordinación institucional actual es deficitaria. Entre Estado, comunidades autónomas y ayuntamientos no existe una coordinación efectiva, como tampoco existe entre los departamentos de vivienda, servicios sociales y sanidad. La experiencia europea se está incorporando progresivamente pero con lentitud. De hecho, por ejemplo, apenas se

${ }^{27}$ Asociación Provivienda: 〈https://provivienda.org〉. org〉. utilizan los fondos europeos para programas de sinhogarismo. El potencial de los fondos europeos es importante para profundizar en políticas de inclusión de las personas sin hogar, tales como: la lucha contra la indiferencia pública y la estigmatización de las personas sin hogar; la protección de las mujeres sin hogar contra la violencia y los abusos; el aumento de los presupuestos nacionales y regionales dedicados a la vivienda social; el trabajo de prevención para evitar el desalojo de viviendas; la intervención en las primeras etapas de la desintegración familiar y la garantía de alojamiento para los jóvenes que abandonan las instituciones; y, por último, la ampliación de la cobertura de la renta mínima de las personas sin hogar.

\section{Discusión}

El análisis sobre las políticas sociales contra el sinhogarismo en el ámbito de la Unión Europea y España plantea cuestiones de importancia para el diseño y efectividad de las políticas públicas. Aquí hacemos referencia a tres.

Una primera cuestión es la referida a cuál es el lugar social e institucional del sinhogarismo, así como su entorno. El sinhogarismo ocupa un espacio casi marginal en las políticas sociales, está en la frontera entre lo visible y lo invisible. Ha sido la crisis financiera del periodo 2010-2014 y la reacción social frente a la pérdida de la vivienda por impago de hipoteca 0 alquiler lo que ha dado visibilidad social a un problema social complejo que comprende desde la población casi invisible que vive en la calle hasta aquellos hogares que por pérdida de ingresos del trabajo han sido expulsados de sus viviendas. Sin embargo, las políticas sociales siguen diferenciando entre la normalidad (población afectada por el impago de una renta e hipoteca y que suelen acumular otros problemas, como desempleo y de integración social) y la marginalidad (los que no tienen una vivienda o lugar donde alojarse). Es la divisoria entre una integración precaria y la exclusión social. Las políticas públicas dan prioridad al primer objetivo y tienden a trasladar al sector social organizado la respuesta y gestión del sinhogarismo con el apoyo de los gobiernos locales. Ha sido en los últimos años cuando las políticas públicas han empezado a adoptar un enfoque global o estratégico del problema del sinhogarismo y, en general, del acceso a la vivienda. En este contexto, el análisis social debe abordar las tensiones y contradicciones entre los discursos políticos y las prácticas institucionales.

Una segunda cuestión de importancia es la aparente paradoja entre el auge de las políticas y estrategias contra la exclusión de un alojamiento o vivienda y el retroceso en el desarrollo de las políticas de vivienda social en casi todos los países miembros de la Unión Europea. La vivienda social existente está siendo objeto de procesos de privatización y se ha producido un estancamiento en su desarrollo. Un 
entorno que nos lleva a señalar que las estrategias contra el sinhogarismo pueden estar condenadas a la inefectividad si no van acompañadas de políticas integrales de vivienda, empleo y servicios sociales, a la vez que ocupan un lugar visible en el conjunto de las políticas sociales y un compromiso financiero correspondiente.

La tercera cuestión es la referente al análisis comparado de las políticas. La europeización de las políticas sociales de España ha sido una realidad creciente mediante la convergencia de conocimientos, prácticas institucionales y políticas públicas. También se está produciendo en el ámbito de las estrategias y políticas contra el sinhogarismo. Sin duda, el aprendizaje en este ámbito, como en otros es un avance positivo para la mejora del diseño de las políticas, la incorporación de actores sociales e institucionales y para medir su efectividad. Una convergencia que, en el caso del sinhogarismo, supone una progresiva superación de las visiones asistencialistas y paternalistas y su sustitución por apoyos integrales en los que las personas afectadas sean protagonistas. Pero es necesario recordar también que estas políticas son competencia de los Estados miembros. Si a ello añadimos el hecho de la diversidad cultural y la path dependency de las políticas sociales, el resultado es que la europeización de las políticas sociales tiene límites definidos que conllevan relativizar los resultados del análisis comparado sin menoscabo del mutuo aprendizaje. En todo caso, el análisis de las políticas sociales es una exigencia para lograr análisis sociales complejos del desarrollo de los derechos sociales.

En suma, el debate de las políticas sobre sinhogarismo está obligado a un análisis y comprensión que tenga en cuenta los tres factores señalados: la necesidad de un mirada amplia al problema (el acceso a la vivienda en general y su conexión con el empleo y los ingresos), la contradicción entre el auge de las políticas integrales y el entorno privatizador de estas y, por último, la necesidad de analizar las políticas sociales a escala europea teniendo en cuenta la diversidad de regímenes de bienestar. 
ARRIBA, A. y RODRÍGUEZ CABRERO, G. (2018): "Housing Plan in Spain for 2018-2021", European Social Policy Network, Flash Report 2018/58, <http://bit. ly/2Y5mAJY>.

BAPTISTA, I. y MARLIER, E. (2019): Fighting Homelessness and Housing Exclusion in Europe: A Study of National Policies, Bruselas, European Commission, 〈http://bit.ly/2nbbNRa〉.

CABRERA, P. (2012): Estudio personas sin techo. Zaragoza 2012, Zaragoza. Cruz Roja.

COMISIÓN EUROPEA (2010): Propuesta de informe conjunto sobre protección social e inclusión social (2010), COM 25 final, Bruselas, Comisión Europea.

- (2013), Hacia la inversión social para el crecimiento y la cohesión, incluida la ejecución del Fondo Social Europeo 2014-2020, COM 83 final, Bruselas, Comisión Europea.

DUQUE, J.M. (2014): “Políticas de servicios sociales para la atención a personas en situación de exclusión residencial grave. Elementos para un diagnóstico y propuestas estratégicas", Zerbitzuan, № 55 , pp. 35-46.

EAPN (2019): Nota de prensa, 〈http://bit.ly/2MDhMef〉.

FACIAM (2012): 10 Propuestas para una estrategia de erradicación del sinhogarismo, Federación de Asociaciones de Centros para la Integración y Ayuda de Marginados, Madrid.

FEANTSA y ABBÉ PIERRE FOUNDATION (2019): Fourth Overview of Housing Exclusion in Europe 2019, 〈http://bit.ly/2KGLf48〉.

FUNDACIÓN EGUÍA-CAREAGA (2015): Estudio sobre la situación de las personas en situación de exclusión residencia grave en la CAPV 2014. Avance de datos. Febrero 2015, Bilbao,
Fundación Eguía-Careaga.

FUNDACIÓN FOESSA (2018a): Exclusión Estructural e Integración Social. Análisis y Perspectivas, 2018, Madrid, Fundación Foessa.

- (2018b): La vulneración del derecho a vivienda: una mirada a la exclusión residencial, Dossier Foessa, Avance de resultados de la encuesta sobre integración y necesidades sociales 2018 con motivo de la Campaña de Personas sin Hogar ¿Y tú que dices? Di basta-Nadie sin hogar, Madrid, Fundación Foessa.

JESSOULA, M.; NATILI, M., PAVOLINI, E. y RAITANO, M. (2019): ESPN Thematic Report on National strategies to fight homelessness and housing exclusion, Italy, European Social Policy Network (ESPN), Bruselas, European Commission.

KANGAS, O. y KALLIOMAA-PUHA, L. (2019): ESPN Thematic Report on National strategies to fight homelessness and housing exclusion, Finland, European Social Policy Network (ESPN), Bruselas, European Commission .

KVIST, J. (2019): ESPN Thematic Report on National strategies to fight homelessness and housing exclusion, Denmark, European Social Policy Network (ESPN), Bruselas, European Commission.

LEGROS, M. (2019): ESPN Thematic Report on National strategies to fight homelessness and housing exclusion, France, European Social Policy Network (ESPN), Bruselas, European Commission.

LLOBET ESTANY, M. y AGUILAR, M. (2016): “El Housing First. El derecho a la vivienda de los más vulnerables”, Barcelona Societat, № 20, pp. 1-14.

MINISTERIO DE SANIDAD, CONSUMO Y BIENESTAR SOCIAL (2019): Estrategia Nacional de Prevención y 
Lucha contra la Pobreza y la Exclusión Social 2019-2023, 〈http://bit.ly/2KIQXZ9〉.

MUÑOZ, M.; SÁNCHEZ, M y CABRERA, P.J. (2014): VII recuento nocturno de personas sin hogar en la ciudad de Madrid, Ayuntamiento de Madrid, Madrid, 2014, 〈http://bit.ly/2H4OM8X〉.

OOSTVEEN, A. (2019): ESPN Thematic Report on National strategies to fight homelessness and housing exclusion, The Netherlands, European Social Policy Network (ESPN), Bruselas, European Commission.

PARLAMENTO EUROPEO (2014): European Parliament Resolution on an EU Homelessness Strategy, 16 enero 2014, Bruselas, European Parliament.

- (2011): European Parliament Resolution on an EU Homelessness Strategy, 14 septiembre 2011, Bruselas, European Parliament.

PERISTA, P. (2019): ESPN Thematic Report on National strategies to fight homelessness and housing exclusion, Portugal, European Social Policy Network (ESPN), Bruselas, European Commission.

PLEACE, N.; BAPTISTA, I.; BENJAMINSEN, L. y BUSCHGEERTSEMA, V. (2018): Homelessness Services in Europe, Brussels, Feantsa, 〈https://www. feantsaresearch.org/public/user/Observatory/ Feantsa-Studies_08_v02[1].pdf〉.

RODRÍGUEZ CABRERO, G. y MARBÁN GALLEGO, V. (2016): Spain's First Comprehensive National Homelessness Strategy, European Social Policy
Network, Flash Report 2016/25, 〈http://bit ly/2H804sX>.

SALES, A. (2013): Diagnosis 2013. Las personas sin hogar en la ciudad de Barcelona y la evolución de la Red de Atención a Personas sin hogar. Barcelona, Xarxa d'Atenció a Personas Sense Llar (XAPSLL), 〈http://bit.ly/2Vi5QSM〉.

SANZO, L. (2019): La estrategia española de garantía de ingresos, 〈http://bit.ly/2Xk4AM9〉.

SIIS-CENTRO DE DOCUMENTACIÓN E INVESTIGACIÓN (2017): III Estudio sobre la situación de las personas en situación de exclusión residencial grave en la Comunidad Autónoma Vasca, 2016, SIIS, Donostia-San Sebastián, 2017.

SIROVÁTKA, T.; JAHODA, R. y MALÝ, I. (2019): ESPN Thematic Report on National strategies to fight homelessness and housing exclusion, Czech Republic, European Social Policy Network (ESPN), Bruselas, European Commission.

URBÉ, R. (2019): ESPN Thematic Report on National strategies to fight homelessness and housing exclusion, Luxembourg, European Social Policy Network (ESPN), Bruselas, European Commission.

ZIOMAS, D.; KONSTANTINIDOU, D.; CAPELLA, A. y VEZYRGIANNI, K. (2019): ESPN Thematic Report on National strategies to fight homelessness and housing exclusion, Greece, European Social Policy Network (ESPN), Bruselas, European Commission. 